\title{
L'INTONATION DU FRANÇAIS ET DU PORTUGAIS: PHONETIQUE ET PHONOLOGIE
}

Philippe Martin*

\begin{abstract}
RESUMO: A entonação, considerada como sequênciade contornos melódicos localizadas nas sílabas acentuadas, define uma estrutura prosódica associada à estrutura sintática. A estrutura prosólica, submetida às restrições de colisões de acentos, de colisão sintática, de eurythmie e de palavra prosódica longa, a priori válidas para todas as línguas românicas, são aplicadas aos domínios do Português Brasileiro para determinar o mecanismo da produção dos contornos melódicos próprios deste sistema linguístico. A gramática prosódica assim definida leva em consideração os dados experimentais de frases em português lidas por brasileiros.
\end{abstract}

PALAVRAS-CHAVE: entonação; estrutura prosódica; contornos melódicos; gramática prosódica; português brasileiro.

\section{INTRODUCTION}

ue ce soit en lecture silencieuse ou oralisée, à haute voix ou
pendant un discours, toute production orale est nécessairement accompagnée d'une variation musicale, d'une évolution mélodique qui constitue la musique de la phrase. Cette musique est manifestée par des montées et des descentes de hauteur, des accélérations et des ralentissements de rythme, des allongements et des raccourcissements syllabiques, des élévations et des chutes d'intensité. Parfois des variations plus complexes mais aisément identifiables fonctionnent comme des clichés mélodiques, liées à des activités ou des comportements spécifiques des locuteurs.

* Département d'Études Françaises, University of Toronto, Toronto, Canada. 
MARTIN, Philippe. Lintonation du français et du portugais: phonetique et phonologie.

Comme pour tout autre objet à décrire, il faut pouvoir faire un tri de toutes les productions prosodiques, tri qui, comme dans d'autres sciences, peut être purement taxinomique et déterminer ses critères dans les formes des réalisations, les courbes de fréquence etc., ou bien utiliser un principe de classement extérieur à ses manifestations, comme la ou les fonctions linguistiques ou autres qui y seraient attribuées.

Depuis longtemps, on sait que la prosodie, dans ses manifestations mélodiques, rythmiques et accentuelles, est corrélée à l'émotion, l'attitude, l'origine géographique réelle ou simulée du locuteur, voire à ses opinions sociales et politiques, et on peut trouver là des principes de classement possibles.

Dans cet exposé, on tentera de mettre en lumière les fonctions proprement linguistiques de l'intonation, certaines bien connues et documentées comme l'indication de la modalité déclarative ou interrogative de la phrase et la division propos thème de l'énoncé, d'autres plus controversées comme la manifestation d'une structure prosodique, indépendante mais associée à la structure syntaxique. Ces trois fonctions serviront de principe directeur pour l'observation et la description des données. Il faudra à chaque fois distinguer ce qui est phonologique et relève du code linguistique de ce qui est phonétique et constitue des réalisations particulières d'unités phonologiques.

Ce n'est pas toujours là une entreprise facile, qui peut aller à l'encontre de bien des idées reçues. Pour des raisons historiques en effet, le domaine des études de l'intonation a été occupé longtemps par des phonéticiens et ingénieurs de traitement du signal, dont les préoccupations majeures étaient souvent bien éloignées de la phonologie. $\Lambda$ l'inverse, les phonologues d'inspiration fonctionnaliste, sans doute influencés par la graphie des unités linguistiques où elle est peu apparente, n'ont concédé à l'intonation qu'un rôle marginal, allant jusqu'à en nier toute place dans la description linguistique. D'autres, influencés par la syntaxe générative et transformationnelle, ont cru voir dans des tracés expérimentaux de nombreux faits qui confor- 
taient leur thèses phonologiques, mais qui se révèlent souvent, à l'examen, comme des artefacts ou des faits acoustiques mal interprétés.

\section{DIFFICULTES DE L'ANALYSE}

Lintonation a toujours semblé difficile à transcrire et à analyser, au point que les chercheurs dans ce domaine ne peuvent se passer d'instruments de mesure acoustique spécialisés. Une des raisons principales se trouve peut-être dans la quasi absence de notation dans les systèmes d'écriture occidentaux, discrétion justifiée par la redondance apparente de la prosodie dans l'écriture. Par conséquent, tout ce qui est prosodique devrait pouvoir être reconstitué à partir de l'écrit, comme le lecteur reconstitue la séquence des voyelles et consonnes à partir de la transcription orthographique de la phrase. Les réalisation relativement peu satisfaisantes à ce jour des systèmes de synthèse à partir du texte démontre à l'évidence le contraire, non seulement parce que la parole synthétique n'a pas d'âme ni d'émotion (devrait-elle d'ailleurs en avoir?), mais surtout parce que la production d'une intonation de phrase "correcte" (c'est-à-dire satisfaisante) doit avoir accès à la structure syntaxique et parfois sémantique de la phrase, et la dérivation d'une réalisation prosodique satisfaisante relève encore au domaine de la recherche.

\section{PHONETIQUE ET PHONOLOGIE DE L'INTONATION}

La phonétique de l'intonation s'inscrit sur les plans articulatoire, acoustique et perceptif, chacun de ces domaines étant relative à une activité de description phonétique particulière.

Sur le plan du signifié, on attribue (au moins) trois fonctions à l'intonation: l'indication de la modalité déclarative ou interrogative de la phrase, 
MARTIN, Philippe. L'intonation du français et du portugais: phonetique et phonologie.

la division en propos et thème, et l'indication partielle ou totale de la structure syntaxique.

\begin{tabular}{|l|l|}
\hline Manifestations & Fonctions \\
\hline Articulatoire & Modalité \\
$\begin{array}{l}\text { Fréquence de vibration laryngée } \\
\text { Pression sous glottique }\end{array}$ & Déclaration \\
\hline Acoustique & Diverrogation \\
\hline Fréquence fondamentale & \\
\hline Perceptive & Indication de la structure syntaxique \\
Hauteur mélodique & Structure prosodique \\
\hline
\end{tabular}

Table 1: Aspects phonétiques de l'intonation (articulatoires, acoustiques et perceptifs), et fonctions linguistiques essentielles (indication de la modalité, division en propos et thème, indication de la structure)

\section{PHYSIOLOGIE: VIBRATION DES CORDES VOCALES}

La mise en vibration des cordes vocales nécessaire à la génération de sons voisés se fait lors de la phase expiratoire du cycle d'expiration, ce qui bouleverse fortement les durées d'inspiration et d'expiration, qui, en l'absence de phonation, sont sensiblement égales. La production des sons de parole non voisés (fricatives et occlusives) a également lieu lors de l'expiration (à l'exception des clics, utilisés dans un très petit nombre de langues).

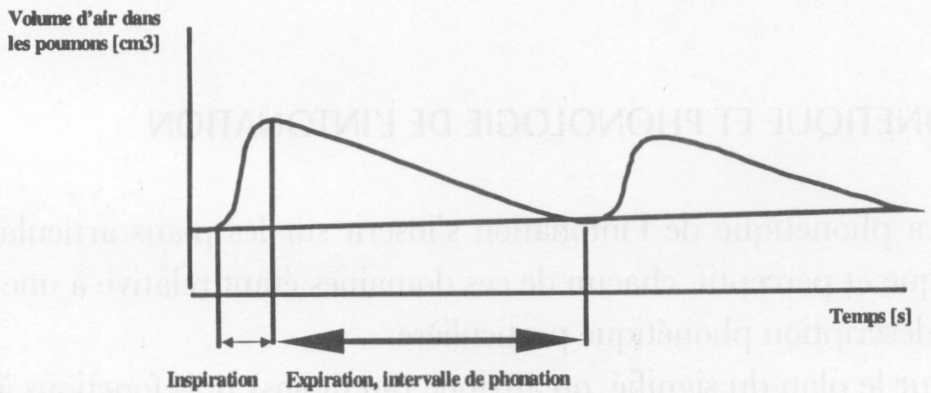

(Fig. 1: Cycle respiratoire en phonation) 
Lintervalle de phonation dépend de l'activité physique, de la condition du locuteur etc. Le cycle respiratoire est évidemment dominant, le tout est d'emmagasiner suffisamment d'air et de gérer l'air disponible en prévision de l'intervalle et de l'effort de phonation prévu.

Ceci est un facteur très important pour l'étude de l'intonation, et détermine les contraintes physiologiques du rythme, la décroissance de la fréquence laryngée etc. La gestion des cycles de respiration (inspiration expiration) déterminent le positionnement des pauses (pauses dites d'inspiration). Le locuteur doit planifier le moment de sa prochaine prise d'air, de manière à le faire coïncider avec une position linguistiquement acceptable (fin de phrase, fin de subordonnée à l'initiale etc.)

Cette gestion dépend de très nombreux facteurs, parmi lesquels:

- L'intensité du son entraîne une plus forte consommation d'air, donc des durées de phonation plus courts, et des intervalles d'inspiration plus longs ou plus "intenses" (et bruyants).

- La vitesse d'élocution: nombre de syllabes émises par unité de temps, consomme plus d'air, donc entraîne des cycles plus courts.

- L'activité de phonation interfère avec d'autres sources de consommation d'air: état émotionnel, fatigue, position du corps etc.

Dans l'apprentissage de l'activité langagière, l'enfant acquiert progressivement la maîtrise de la gestion des paramètres pour

- placer convenablement les pauses d'inspiration (de respiration) aux endroits syntaxiquement corrects;

- obtenir une intensité des sons émis relativement constante du début à la fin de chaque cycle phonatoire.

Ceci est à l'origine de la ligne de déclinaison, correspondant à la tendance observée quant à une chute progressive de la fréquence laryngée du début à la fin d'un cycle phonatoire. 
MARTIN, Philippe. L'intonation du français et du portugais: phonetique et phonologie.

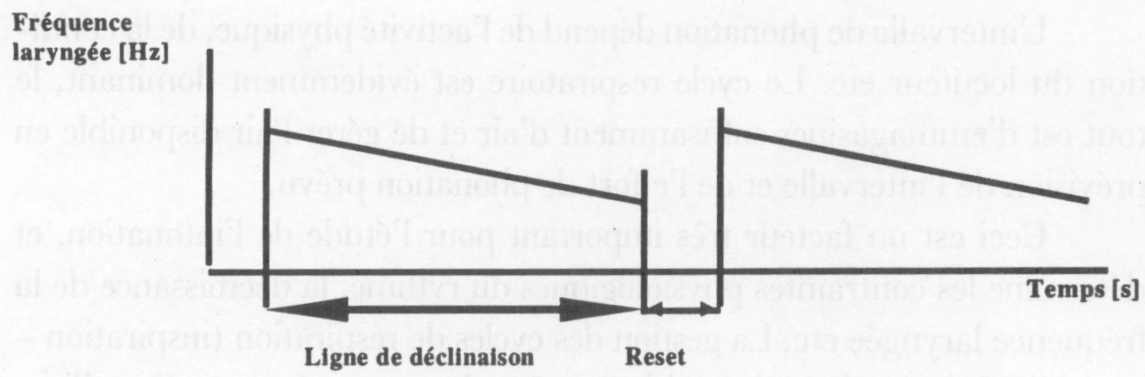

(Fig. 2: Ligne de déclinaison de Fo et reset)

Diverses études physiologiques (coupes, rayons X, scanner, laryngoscopie, modélisation,...) ont mis en lumière les détails du mécanisme qui permet la vibration des cordes vocales. Celui-ci détermine un positionnement particulier des cordes vocales de manière à permettre l'ouverture progressive du passage de l'air des poumons à l'expiration, ouverture suivie d'une fermeture brusque due à la combinaison une chute de pression (expliquée par l'effet Bernoulli).

L'observation du mécanisme phonatoire montre également le lien généralement présent entre la fréquence de vibration laryngée $\left(F_{1}\right)$ et l'intensité. En simplifiant, on peut dire que la fréquence dépend de la tension des cordes vocales (contrôlée par plusieurs muscles, principalement le cricothyoïdien, le thyro-arytenoïde interne et le crico-aryténoïde latéral, cf. Lieberman, 1977), et de la pression d'air sous la glotte au moment de la phonation (la pression sous glottique, $\mathrm{p}_{\mathrm{s}}$ ), pression dont dépend aussi l'intensité. L'augmentation de $\mathrm{p}_{\mathrm{s}}$ provoque donc à la fois celle de l'intensité et celle de la fréquence laryngée.

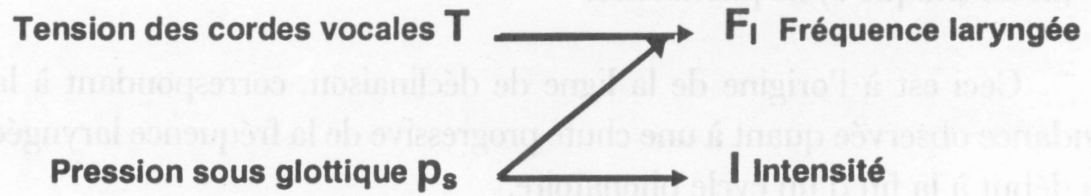


$\Lambda \mathrm{P}_{\mathrm{s}}$ constant, l'intensité est constante et la fréquence laryngée dépend de T. $\Lambda$ tension T constante, l'intensité et la fréquence laryngée dépendent de $\mathrm{p}_{\mathrm{s}}$.

Des valeurs typiques de fréquence laryngée sont:

Femmes: $\quad 150-300 \mathrm{~Hz}$ (période laryngée: $7 \mathrm{~ms}-3 \mathrm{~ms}$ )

Hommes: $\quad 90-200 \mathrm{~Hz}(11 \mathrm{~ms}-5 \mathrm{~ms})$

Enfants: $\quad 250-400 \mathrm{~Hz}(4 \mathrm{~ms}-2.5 \mathrm{~ms})$

Bébés: $\quad 300-800 \mathrm{~Hz}(3 \mathrm{~ms}-1.2 \mathrm{~ms})$

\section{LA COURBE MELODIQUE}

La vibration des cordes vocales lors de la phonation entrâne la génération d'harmoniques, dont l'analyse acoustique (par transformée de Fourier ou filtrage) permet la mesure de la fréquence fondamentale, qui correspond à la première harmonique et aussi à l'intervalle de fréquence entre deux harmoniques consécutives. La fréquence fondamentale Fo constitue une estimation de la fréquence de vibration laryngée, plus ou moins proche de ce que donnerait une mesure directe sur les vibrations des cordes vocales, et susceptible de s'en écarter de par les propriétés mathématiques du procédé d'analyse spectrale utilisé. Linsi, le fenêtrage inhérent au spectre acoustique se traduit par une perte de résolution temporelle.

D'autre part, les conditions d'enregistrement de la parole analysée ne respectent pas toujours les conditions nécessaires à l'analyse. La présence de bruits d'origines diverses, de filtrages et de déphasages lors de l'enregistrement peuvent rendre la mesure de Fo inexacte ou imprécise. La première harmonique peut être absente ou indécelable par la présence de filtrage ou de bruits de basse fréquence, les harmoniques successives peuvent appartenir à des sources différentes, celle de la voix analysée et une source de bruit etc. D'une manière peu évidente, des déphasages intem- 
MARTIN, Philippe. L'intonation du français et du portugais: phonetique et phonologie.

pestifs d'harmoniques dus aux amplificateurs d'enregistreur à cassette peuvent également perturber la mesure,

Toutefois, des logiciels modernes tels WinPitch (WinPitch, 1996) mettent en cuvre des algorithmes suffisamment résistants aux bruits pour assurer l'affichage de courbes mélodiques en temps réel avec une fiabilité excellente. Les algorithmes de détection de la fréquence fondamentale utilisent différentes informations en parallèle en assurent un tri optimum du signal utile et du bruit.

La mise en vibration des cordes vocales a lieu lors de la phonation des sons de parole dits voisés. La courbe mélodique, ou courbe de fréquence fondamentale, n'aura donc pas de valeur pour les sons non voisés (occlusives et fricatives sourdes). Conventionnellement, c'est la valeur zéro qui est affichée. Les vides ou "trous" de la courbe mélodique correspondent donc aux sons sourds ou aux silences.

D'autre part, certaines consonnes voisées sont émise avec une chute de pression sous glottique, entraînant une baisse de Fo, ce qui se traduit, entre deux voyelles, par un mouvement microprosodique en forme de "V". C'est le cas des occlusives [b], [d], [g] et des fricatives [v], [z], [3], ainsi que des nasales [n] et [D], et [r] intervocalique. Par contre, [1], les nasales $[\mathrm{m}],[\mathrm{n}]$ ainsi que $[\mathrm{r}]$ en position finale de provoquent pas de micro perturbation dans la ligne mélodique.

Ces variations microprosodiques peuvent servir de critères pour une première segmentation de la courbe mélodique.

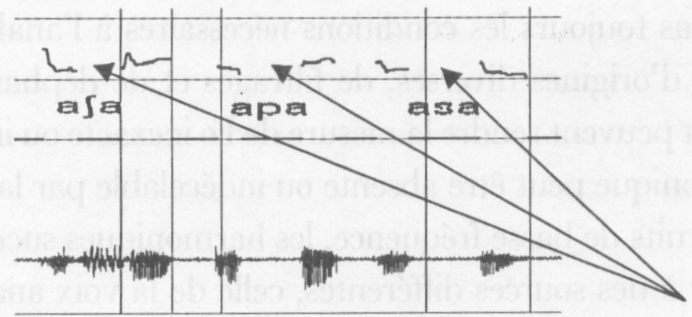

(Fig. 3: Interruption de la courbe mélodique par des consonnes sourdes) 


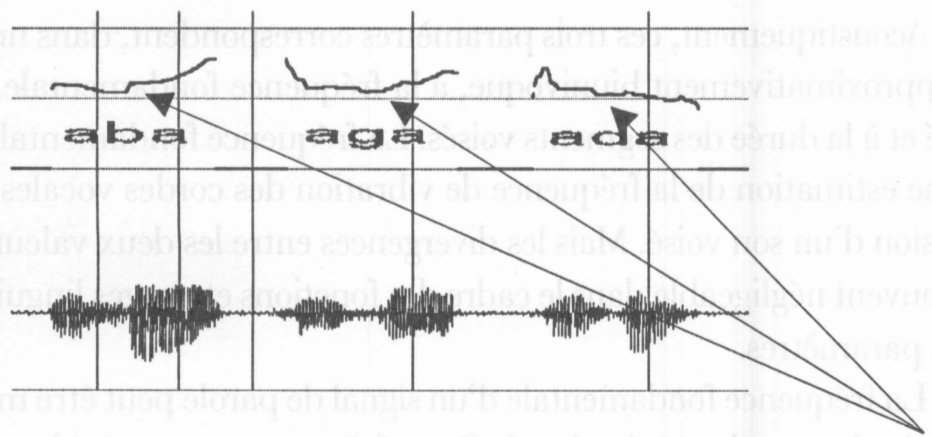

(Fig. 4: Chute locale de la courbe mélodique par des consonnes voisées)

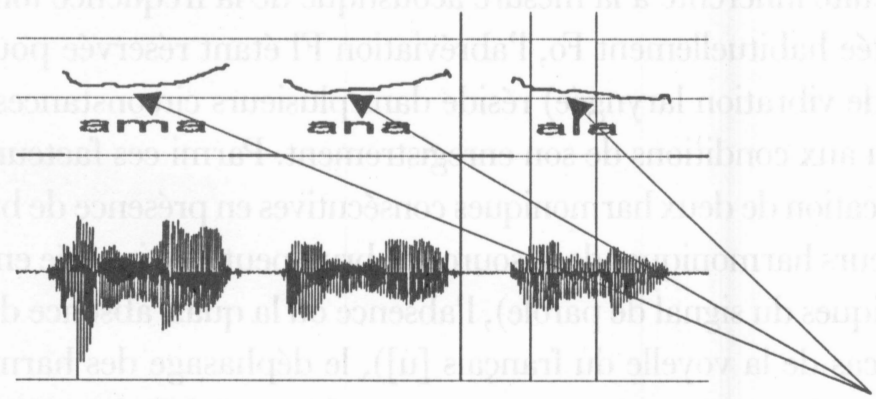

(Fig. 5: Continuité de la courbe mélodique par des consonnes voisées)

\section{LES PARAMETRES ACOUSTIQUES DE L'INTONATION}

$\mathrm{Si}$ on attribue plusieurs fonctions et usages à l'intonation, les faits prosodiques relevant de l'observation acoustique présents dans l'énoncé combineront les événements relatifs à chacun de ces fonctions et usages. Traditionnellement, et en opposition avec les caractéristiques acoustiques des éléments segmentaux (les phonèmes), on sépare les spectres, dont les caractéristiques relèvent de la configuration du conduit vocal lors de la phonation, des faits déterminés par la source de la génération du son, c'est à dire la fréquence laryngée, l'intensité et la durée de l'émission vocalique. 
MARTIN, Philippe. L'intonation du français et du portugais: phonetique et phonologie.

Acoustiquement, ces trois paramètres correspondent, dans une relation approximativement biunivoque, à la fréquence fondamentale, à l'intensité et à la durée des segments voisés. La fréquence fondamentale est en fait une estimation de la fréquence de vibration des cordes vocales lors de l'émission d'un son voisé. Mais les divergences entre les deux valeurs est le plus souvent négligeable dans le cadre des fonctions et usages linguistiques de ces paramètres.

La fréquence fondamentale d'un signal de parole peut être mesuré à partir des harmoniques du signal. Cette fréquence correspond en effet à l'intervalle existant entre deux harmoniques consécutifs dans les spectre. La difficulté inhérente à la mesure acoustique de la fréquence fondamentale (notée habituellement Fo, l'abréviation Fl étant réservée pour la fréquence de vibration laryngée) réside dans plusieurs circonstances liées au signal ou aux conditions de son enregistrement. Parmi ces facteurs, citons l'identification de deux harmoniques consécutives en présence de bruit (une ou plusieurs harmoniques de la source de bruit peut être insérée entre deux harmoniques du signal de parole), l'absence ou la quasi absence d'harmoniques (cas de la voyelle du français [u]), le déphasage des harmoniques (les harmoniques mesurées ne se correspondent pas dans le temps, certaines arrivant avant d'autres dans le spectre par distorsion de filtrage), l'absence de première harmonique etc. [Martin, 1983, Hess, 1981].

Depuis quelques années, les méthodes basées sur l'analyse spectrale en composante harmonique du signal de parole sont dominantes. Les temps de calcul, autrefois rédhibitoires, sont devenus raisonnables au point de pouvoir opérer en temps réel sur un ordinateur personnel [WinPitch, 1996]. Dans des conditions des bruits et de distorsions modérées à sévères, les algorithmes de détection de Fo à base spectrale sont suffisamment fiables pour réaliser des analyses de données de grands corpus très rapidement. Ceci devrait changer à court terme les conditions de recherche en prosodie, qui ont été longtemps difficiles de part les équipements nécessaires et la formation technique des opérateurs. 


\section{MODALITE}

La fonction la plus connue et la plus documentée de l'intonation est celle qui assure l'indication de la modalité de la phrase. Posons que la modalité caractérise le rapport existant entre le locuteur et l'auditeur au moment de la prise de parole par le locuteur. Toutes sortes de rapports peuvent être envisagés a priori. Par exemple: déclaration / souhait / demande / ordre / désir / ...

Une grille très fines définissant ce rapport peut être envisagée. Par exemple, dans certains systèmes linguistiques, le rapport de supériorité, d'égalité, d'infériorité sociale peut être codé dans la morphologie du verbe, des pronoms personnels etc. En français les pronoms tu / vous jouent ce rôle. En anglais, ce rapport se traduit par l'utilisation du pronom you opposé à celui du prénom de l'auditeur etc. Des études récentes (Cresti, E., Martin, Ph., Moneglia, M., 1998) proposent des descriptions des variantes de modalité, inscrites dans le cadre général de l'acte d'énonciation.

Pour simplifier, disons que la modalité traduit l'attitude fondamentale du locuteur dans la relation de communication. En se laissant guider par les variantes de signifiant (morphologie des verbes par exemple), on dira que la modalité correspond à l'attitude fondamentale du locuteur qui soit entrée dans le système de la langue. Ainsi en français, la morphologie du verbe semble nous indiquer qu'il existe trois modalités: la déclaration, l'interrogation et l'ordre, que l'on retrouve dans les exemples:

Déclaration:

tu viens

Interrogation:

tu viens?

Ordre:

tu viens ! 
MARTIN, Philippe. L'intonation du français et du portugais: phonetique et phonologie.

Ces différentes modalités peuvent également être encodées par un mouvement mélodique, donc par l'intonation. En utilisant des exemples à deux syllabes, dont la dernière est accentuée, ce mouvement mélodique est facile à localiser.

Déclaration: tu viens. réponse à la question "que dois-je faire?"

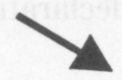

Interrogation: tu viens? réponse à la déclaration "je fais qque chose ce soir"

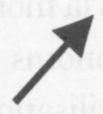

Ordre: tu viens! réponse à "que dois-je faire?"

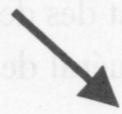

Il y aurait donc 3 contours mélodiques corrélatifs de la modalité de l'énoncé: un contour descendant déclaratif, un contour montant interrogatif, et un contour fortement descendant indiquant l'ordre.

Guidé par les différences (ou le peu de différence) entre signifiés, on est conduit à considérer l'ordre comme une variante de la déclaration, variante dont le signifiant mélodique ne peut être neutralisé par un changement de forme verbale.

En fait, les trois modalités traditionnelles déclaration / interrogation/ ordre proviennent d'une typologie basé sur la syntaxe et la morphologie des verbes. Même de ce point de vue, on s'aperçoit que les formes de l'impératif sont empruntées à d'autre modes: en français, au présent de l'indicatif.

Dans ces exemples, la modalité est indiquée par un trait syntaxique d'inversion et le contour mélodique des neutralisé de ce point de vue. II peut donc être réalisé comme montant, plat ou descendant. 
Le contour impératif descendant se trouve nécessairement neutralisé, et apparaît dès lors comme une variante du contour de modalité déclaratif.

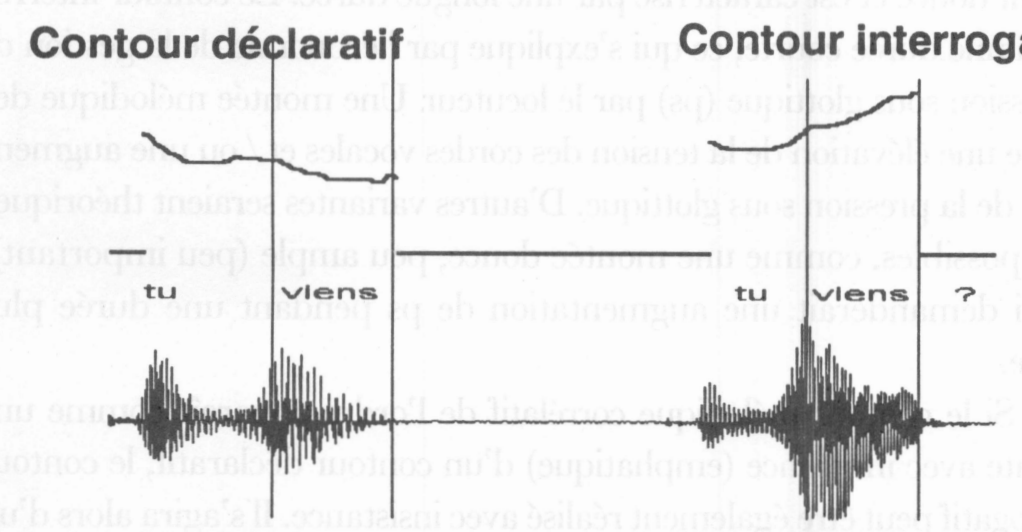

(Les contours correspondent aux variations mélodiques entre les deux barres verticales)

\section{INSISTANCE - IMPLICATION}

Nous avons donc deux contours de modalité, un contour descendant déclaratif, et un contour montant interrogatif. Le contour descendant impératif est alors considéré comme une variante du contour déclaratif, variante avec insistance éventuelle.

Du point de vue de la description phonologique, on a donc une opposition entre deux contours de modalité montant / descendant. Si on décide d'adopter le trait montant comme marqué nous avons donc le système simple:

Déclaratif

Interrogatif
Cd

$\mathrm{Ci}$
[ - Montant]

[+ Montant] 
MARTIN, Philippe. L'intonation du français et du portugais: phonetique et phonologie.

Du point de vue phonétique, ces contours se placent, en français, sur la seule syllabe accentuée de l'énoncé, plus précisément sur la voyelle et sur la consonne voisée qui suit si elle existe.

Le contour déclaratif est réalisé par une descente mélodique relativement douce et est caractérisé par une longue durée. Le contour interrogatif a une durée courte, ce qui s'explique par l'économie de la gestion de la pression sous glottique (ps) par le locuteur. Une montée mélodique demande une élévation de la tension des cordes vocales et / ou une augmentation de la pression sous glottique. D'autres variantes seraient théoriquement possibles, comme une montée douce, peu ample (peu important), ce qui demanderait une augmentation de ps pendant une durée plus longue.

Si le contour mélodique corrélatif de l'ordre apparaît comme une variante avec insistance (emphatique) d'un contour déclaratif, le contour interrogatif peut être également réalisé avec insistance. Il s'agira alors d'un contour corrélatif de la surprise.

On a donc les réalisations emphatiques, avec insistance, suivantes:
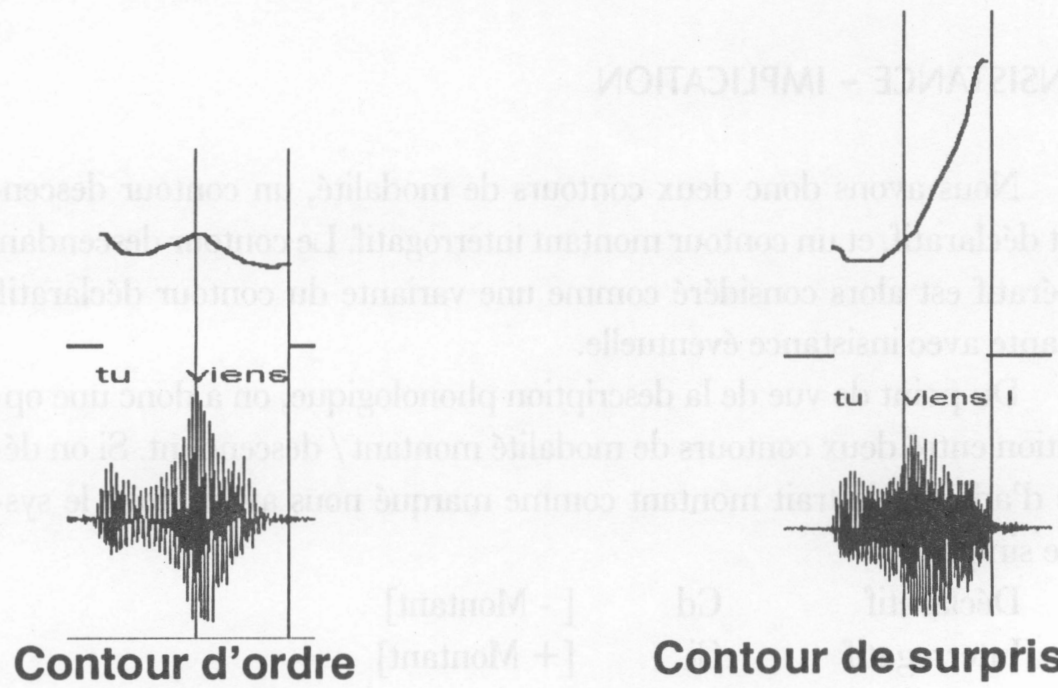

Contour d'ordre

Contour de surprise 
Rev. $A N P O L L$, n. 6/7, p. 255-295, jan./dez. 1999

Phonétiquement, les contours mélodiques correspondant se réalisent par une chute plus grande de mélodie dans le cas déclaratif (ordre), et par une montée plus ample dans le cas interrogatif (surprise). En utilisant le trait phonologique $\Lambda$ mple (dénotant une variation plus forte de mélodie) pour en rendre compte, on a

\begin{tabular}{|l|c|c|c|c|}
\hline & Déclaratif & Interrogatif & Ordre & Surprise \\
\hline & $\mathrm{Cd}$ & $\mathrm{Ci}$ & $\mathrm{Co}$ & $\mathrm{Cs}$ \\
\hline Montant & - & + & - & + \\
\hline Ample & - & - & + & + \\
\hline
\end{tabular}

$\Lambda$ côté de l'insistance, l'emphase portant sur l'énoncé, on peut également envisager l'insistance portant sur le contexte existant au moment de l'énonciation. Le contexte contient toutes les informations transmises au moyen du langage avant l'énoncé considéré, la situation correspond aux informations non linguistiques présentes dans l'acte de parole.

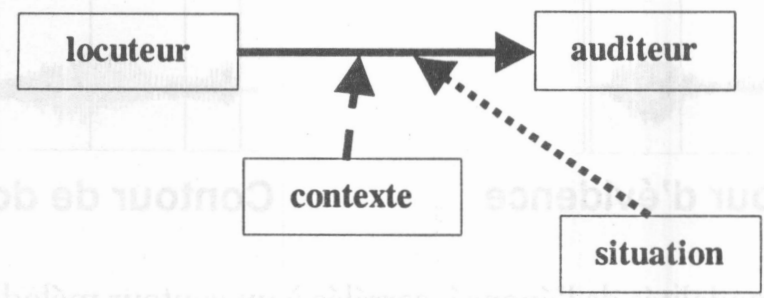

Linsistance portée sur le contexte, contenant les informations apportées par la première phrase, est indiquée par un mouvement mélodique particulier, présentant phonétiquement une bosse au cours de la descente mélodique.

Ex. "c'est Jean qui est venu?" "oui." 
MARTIN, Philippe. L'intonation du français et du portugais: phonetique et phonologie.

Ce contour particulier est corrélatif de l'évidence, qui apparaît ainsi comme une déclaration avec emphase sur le contexte. De même, l'emphase sur le contexte existe dans le cas interrogatif, et est également marquée phonétiquement par un mouvement montant descendant placé à la fin de la montée mélodique d'interrogation. Il s'agit alors d'un contour dénotant le doute, qui apparaît comme une interrogation avec insistance sur le contexte.

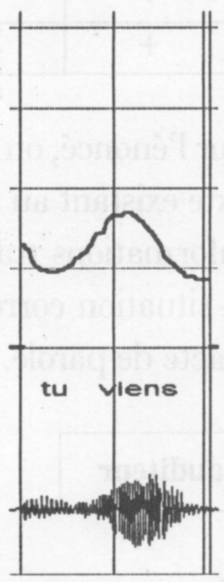

Contour d'évidence

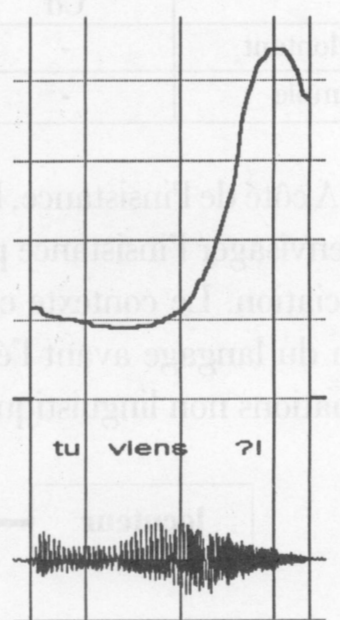

Contour de doute

Les 6 modalités de l'énoncé, corrélée à un contour mélodique spécifique, sont donc:

\begin{tabular}{|l|l|l|}
\hline- & Insistance & Insistance contexte \\
\hline Déclaration & Ordre & Evidence \\
\hline Interrogation & Surprise & Doute \\
\hline
\end{tabular}

La description phonologique de ces contours de modalité utilise par exemple les traits de pente (montant / descendant), d'amplitude de variation mélodique (ample / restreinte), de présente d'une forme montante 
Rev. $A N P(O L L$, n. 6/7, p. 255-295, jan./dez. 1999

descendante ("cloche").Si on adopte les traits Montant, $\Lambda$ mple et Cloche comme traits marqués, la description des 6 contours de modalité sont:

\begin{tabular}{|l|c|c|c|c|c|c|}
\hline & Déclaratif & Interrog. & Ordre & Surprise & Evidence & Doute \\
\hline Montant & - & + & - & + & - & + \\
\hline Ample & - & - & + & + & & \\
\hline Cloche & - & - & - & - & + & + \\
\hline
\end{tabular}

\section{PROPOS THEME - CONTRAINTES}

La division prosodique de la phrase en thème et propos a été souvent décrite et est bien documentée. Dans une des formes que prend cette division, le thème peut être défini comme la deuxième partie de la phrase qui suit le propos, et qui reprend des informations déjà connues dans le processus d'énonciation. Ninsi, à la question que fais Jules à Aix ?, la réponse il enseigne à Aix avec un contour mélodique descendant sur la dernière syllabe accentuée du propos enseigne et un contour plat sur la dernière syllabe accentuée du thème à $A i x$ réalise une division de la phrase qui implique qu'en effet, la partie thème contient des informations déjà connues, et simplement rappelées dans la phrase.

En discours spontané, la partie thème renvoie souvent à un élément du contexte présent dans la situation de communication, mais pas nécessairement énoncée explicitement. Un énoncé aussi courant que bonjour madame est souvent accompagné d'un contour mélodique divisant la phrase en propos (bonjour) et thème (madame), le référent du thème étant déjà connue par la situation ou le contexte. Si la situation telle qu'appréciée par le locuteur ne contient pas cette information, les contours mélodiques seront cette fois montant et descendant, marquant l'absence d'une division propos thème (cf. Deuxième exemple bonjour les dégâts). 
MARTIN, Philippe. L'intonation du franģais et du portugais: phonetique et phonologie.
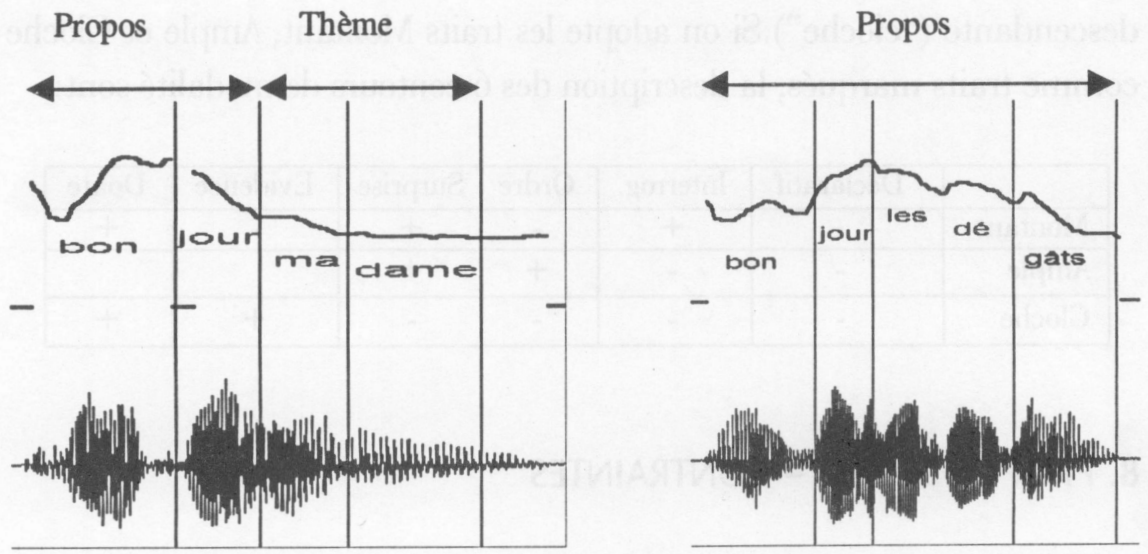

Les variétés interrogatives présentent les mêmes caractéristiques, mais cette fois le deuxième contour finissant le thème sera montant.
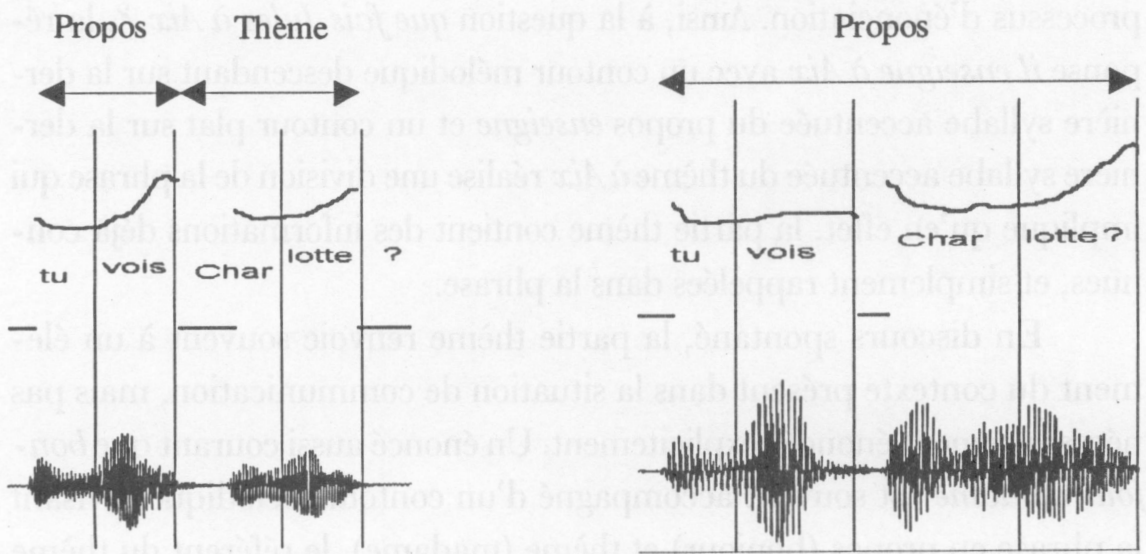

Dans le premier tracé, le pronom tu est coréférent de Charlotte (équivalent de Charlotte, est-ce que tu vois [cette maison là-bas]?), dans le second Charlotte est objet de tu vois (équivalent de est-ce que c'est Charlotte que tu vois?). 
Rev. $A N P() L L$, n. 6/7, p. 255-295, jan./dez. 1999

\section{STRUCTURE PROSODIQUE ET SYNTAXE - CONTRAINTES}

Le concept de structure prosodique repose sur l'hypothèse suivante: il existe, indépendamment de toute autre structure impliquant des unités du discours et de la phrase, une structure qui organise hiérarchiquement des unités minimales prosodiques (des mots prosodiques), liées aux syllabes accentuées de la phrase. Partant, cette structure est indiquée par un mécanisme impliquant des marqueurs existant dans les mots prosodiques.

Les parties perceptivement les plus importantes dans la phrase étant les syllabes accentuées, et la phrase la plus simple possible étant composée d'un mot unique d'une seule syllabe, nécessairement accentuée, l'unité minimale prosodique ou mot prosodique apparaît comme une unité de nature prosodique comportant un et un seul segment correspondant à une syllabe accentuée. Il y a donc autant de mots prosodiques dans une phrase que de syllabes accentuées, d'autres manifestations prosodiques comme les accents d'insistance et d'emphase étant donc surajoutées au mot prosodique.

$\mathrm{Si}$ une telle structure organise les mots prosodiques, il existe nécessairement un mécanisme, de nature prosodique, qui en assure l'indication sous la forme de marqueurs à déterminer. Une fois de plus, un mot prosodique pouvant correspondre à une seule syllabe, ce marqueur peut se retrouver dans le segment prosodique relatif à cette syllabe accentuée, dans les manifestations prosodiques de l'accent, mais éventuellement aussi dans des positions nécessairement repérables même si le mot est réduit à une seule syllabe, c'est-à-dire le début et la fin du mot prosodique.

En observant les manifestations de ces marqueurs à l'intérieur d'une structure prosodique connue, on doit pouvoir en déterminer le fonctionnement, et donc la grammaire prosodique de la phrase.

Reste alors à déterminer la structure prosodique d'une phrase donnée dans une énonciation donnée. Les techniques habituelles cherchent à prendre en quelque sorte la structure syntaxique en défaut pour forcer la structure prosodique à se manifester. Elles utilisent des phrases syntaxi- 
MARTIN, Philippe. L'intonation du français et du portugais: phonetique et phonologie.

quement ambiguës (type un joueur de football américain), des phrases dépourvues de marqueurs syntaxiques (énumérations avec hiérarchie, comme les numéros de téléphone). La lecture oralisée est également de nature à révéler l'organisation prosodique de la phrase, le style de la lecture consistant précisément à réaliser une adéquation entre syntaxe et intonation. En discours spontané, on n'a évidement pas accès à la structure prosodique de manière aussi sûre.

Le résultat de cette recherche portant sur les énoncés lus permet de découvrir, pour le français, les mécanismes suivants, partant des configurations simples aux structures plus complexes, mais en se limitant ici au seul propos, donc au phrases dépourvues de thème:

1. Une phrase à une seul mot prosodique n'a pas de structure prosodique, et le contour placé sur la seule syllabe accentuée nindique donc que la modalité;

2. Une phrase à deux mots prosodiques ne présente qu'une seule structure possible: la première syllabe accentuée du mot qui ne porte pas le contour de modalité ne peut que se différencier de celui-ci;

3. Avec trois mots prosodiques, trois configurations sont possibles:

a)

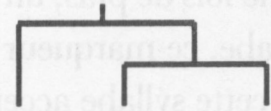

Antoine adore les pommes

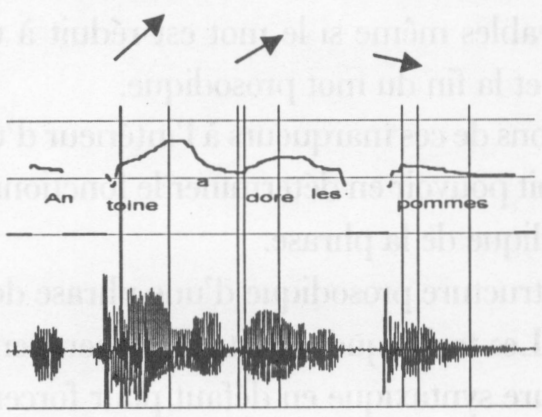

Au contour de modalité final descendant placé sur pommes s'opposent, en deux niveaux différents, le contour montant de la syllabe accentuée de Antoine, et le contour montant, mais de moindre amplitude de variation, placé sur la syllabe accentuée de adore.

La structure prosodique est ici congruente à la structure syntaxique. 
b)

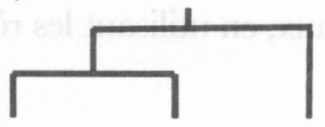

Antoine adore les pommes
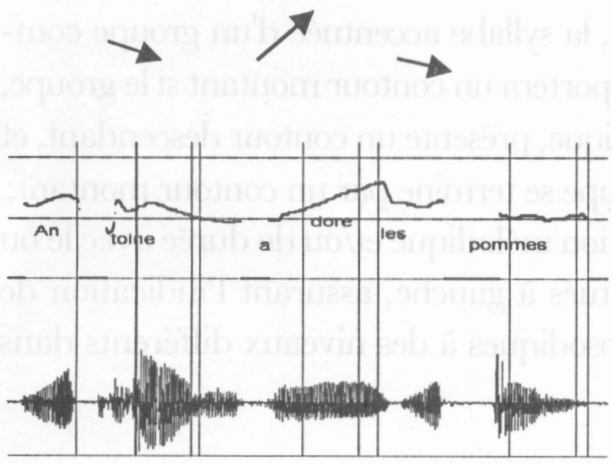

c)

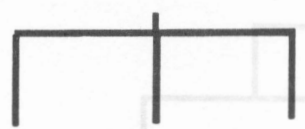

Antoine adore les pommes

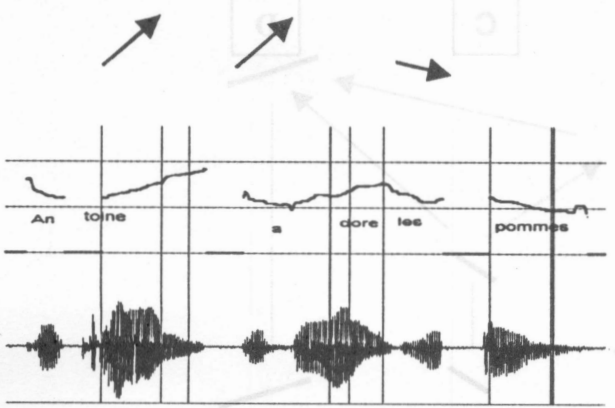

Au contour de modalité final descendant placé sur pommes s'oppose le contour montant sur la syllabe accentuée de adore. A ce contour montant s'oppose un contour descendant placé à sa gauche et sur la syllabe accentuée de Antoine.

La structure prosodique organise ici les unités prosodiques différemment de celle qui est réalisée par la structure syntaxique pour les unités correspondantes aux mots prosodiques.
Au contour de modalité final descendant placé sur pommes s'opposent les contours montants sur la syllabe accentuée de Antoine et sur celle de adore.

La structure prosodique est ici celle d'une énumération. 
MARTIN, Philippe. L'intonation du français et du portugais: phonetique et phonologie.

Ce mécanisme de base se généralise à un nombre plus grand de mots prosodiques organisés en plusieurs niveaux, en utilisant les règles de contour suivantes:

- contraste de pente mélodique envers le contour de droite terminant un groupe prosodique donné. Linsi, la syllabe accentuée d'un groupe comprenant deux mots prosodiques portera un contour montant si le groupe, et donc le deuxième mot prosodique, présente un contour descendant, et un contour descendant si le groupe se termine par un contour montant;

- contraste d'amplitude de variation mélodique et/ou de durée avec le ou les contours de même pente situés à gauche, assurant l'indication de l'appartenance des groupes prosodiques à des niveaux différents dans la structure.

En examinant les mécanismes d'expansion de la structure prosodique, on conclut facilement que la grammaire de la structure prosodique indique une dépendance à droite: un contour donné dépend du contour qui le domine situé à sa droite. Schématiquement, ce mécanisme, peut se représenter de la manière suivante:

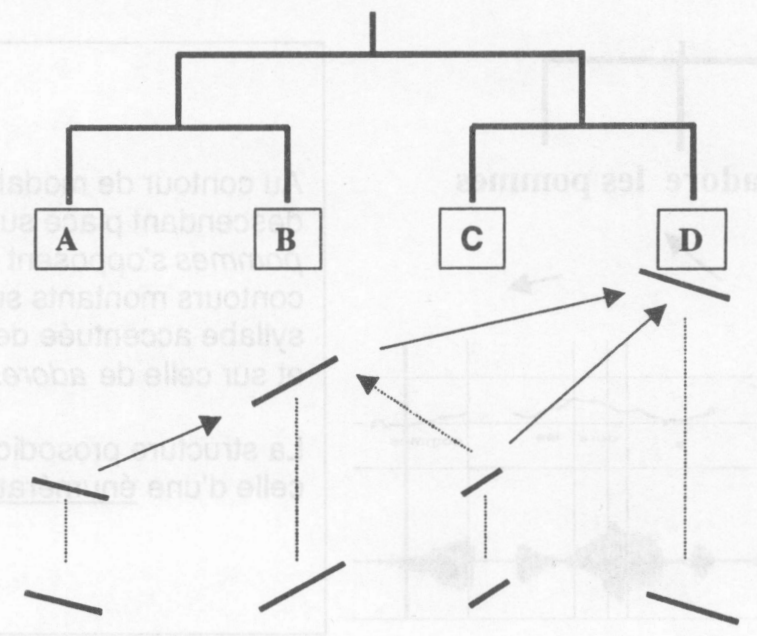


Les flèches en trait plein indiquent un contraste de pente mélodique marquant la dépendance à droite du contour, celle en pointillé un contraste d'amplitude de variation mélodique. On voit que par expansion le contour final d'un groupe prosodique se transmet toujours à droite: c'est le mot prosodique terminal du groupe qui hérite du contour dominant à chaque niveau de la division d'un groupe.

Tout comme les autre unités phonologiques, les contours prosodiques sont susceptibles d'être neutralisés dans leur fonctionnement, et, partant, voir leur réalisation modifiée. C'est le cas en particulier de l'avant dernier contour d'une phrase déclarative, qui, dans sa fonction d'indication de la structure prosodique, est toujours montant puisqu'il s'oppose à un contour à droite nécessairement descendant. C'est effectivement ce qu'on observe, aussi bien dans les corpus lus que les productions spontanées: l'avant dernier contour y est montant, plat ou descendant.

\section{ASSOCIATION PROSODIE - SYNTAXE}

\subsection{COLLISION SYNTAXIQUE}

S'il apparaît que la solution syntaxique d'énonciation de l'exemple

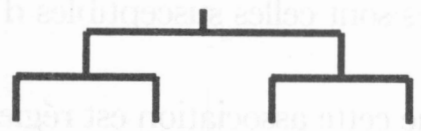

Le frère de Max a mangé les tartines
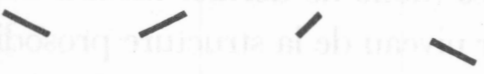
MARTIN, Philippe. L'intonation du français et du portugais: phonetique et phonologie.

n'est pas la seule possible, puisqu'on peut toujours énoncé cette phrase comme une énumération (comme en déclamation poétique par exemple):

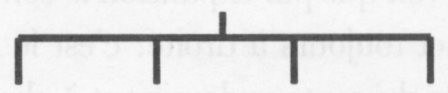

Le frère de Max a mangé les tartines
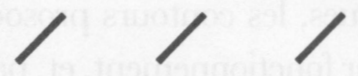

Mais on constate aussi que n'importe quelle structure ne peut être associée à cette phrase, comme

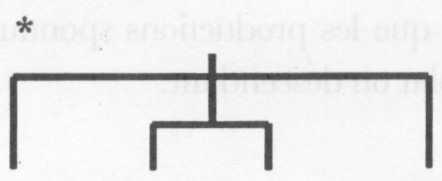

Le frère de Max a mangé les tartines

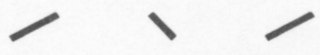

Le problème est donc de déterminer quelles sont parmi toutes les structures possibles déterminées par analyse combinatoire ( $3 \mathrm{SP}$ pour 3 mots prosodiques, $11 \mathrm{SP}$ pour 4 etc.), celles qui pourront être associées à une structure syntaxique donnée, et accessoirement, parmi toutes les structures que l'on peut associer à la SS, quelles sont celles susceptibles d'être favorisées par le locuteur.

On peut montrer (Martin, 1987) que cette association est régie par la condition de collision syntaxique, qui empêche deux unités syntaxiques correspondant à des mots prosodiques (donc de dernier niveau dans la structure) d'être regroupée au dernier niveau de la structure prosodique. Une structure prosodique telle que ne peut donc être associée à la structure 
syntaxique de cet exemple puisque les unités (de Max) et (a mangé) se retrouvent dans le groupe prosodique [de Max a mangé].

\section{[Le frère [de Max a mangé] les tartines]}

Cette condition se décrit par une contrainte de collision syntaxique, qui interdit à la structure prosodique de regrouper deux mots prosodiques dont les unités syntaxiques correspondantes sont dominés par des nœuds différents.

Contrainte de collision syntaxique: * $(\Lambda \ldots .].[\mathrm{N})(\mathrm{M}][\ldots \mathrm{Z})$

$\mathrm{N}$ et $\mathrm{M}$ sont des mots prosodiques dont les correspondants syntaxiques appartiennent à des groupes différents, et ne peuvent être réunies dans un même groupe prosodique [MN].

\subsection{COLLISION D'ACCENT}

Une autre contrainte, celle-là bien connue, concerne l'impossibilité d'avoir deux syllabes accentuées successives, à moins qu'elles ne soient séparées par une pause ou une combinaison de segments consonantiques qui assurent suffisamment de distance temporelle entre les deux accents. En cas de collision, l'accent de la première syllabe concernée recule sur la syllabe immédiatement à droite, ou sur celle normalement occupée par l'accent secondaire. En français, l'accent recule ou disparaît si aucune syllabe a droite ne peut accueillir l'accent déplacé. En voici quelques exemples:

Un soulier noir déplacement du premier accent 
MARTIN, Philippe. L'intonation du français et du portugais: phonetique et phonologie.

Un soulier (pause) noir pas de déplacement $\mathbf{X} \mathbf{X}$

Une tomate verte pas de collision du fait de la séquence $\mathbf{x} \mathbf{x}$ consonantique longue [tv] entre les deux accents

Un chien noir

$\mathbf{X}$

désaccentuation de la première syllabe impliquée

dans la collision

\subsection{LONGUEUR DU MOT PROSODIQUE}

La contrainte de longueur du mot prosodique porte sur le nombre maximum de syllabes inaccentuées successives. Cette contrainte est spécifique au français qui permet la désaccentuation de syllabes portant normalement l'accent tonique. Les valeurs expérimentales oscillent autour de 6 ou 7, la longueur maximum du mot prosodique (qui se termine nécessairement en français par une syllabe accentuée) dépendant de la vitesse d'élocution (nombre de syllabes émises par seconde).

\subsection{EURYTHMIE}

La contrainte d'eurythmie est basée sur la tendance constatée empiriquement à réaliser la durée de groupes syntaxiques de manière sensiblement égale, soit en regroupant les unités de manière à obtenir ces durées égales, soit en modulant la durée des syllabes dans des groupes au nombre de syllabes différents de manière à obtenir le même effet. (cf. 
Wolland, 1985). Les mots et les groupes prosodiques auront donc tendance à avoir les mêmes durées totales à leurs niveaux respectifs dans la structure.

Si on examine les exemples en supposant que la deuxième condition est remplie, c'est-à-dire qu'il y tendance à regrouper les unités de manière à obtenir un nombre de syllabes sensiblement égal, syllabes qui ont donc des durées semblables, la tendance au regroupement des unités de

Arthur mangeait des poissons délicieux

$\begin{array}{lllllllllll}\mathbf{X} & \mathbf{x} & \mathbf{x} & \mathbf{x} & \mathbf{x} & \mathbf{x} & \mathbf{x} & \mathbf{x} & \mathbf{x} & \mathbf{x}\end{array}$

est donc

[Arthur mangeait] [des poissons délicieux]

4 syll. 6 syll.

de manière à mieux équilibrer le nombre de syllabes des groupes prosodiques [Arthur mangeait] [des poissons délicieux], par rapport au groupement syntaxique

[Arthur] [mangeait des poissons délicieux]

2

8

Par contre, si l'adjectif "délicieux" est remplacé par "rouges", la solution congruente à la syntaxe apparaît moins déséquilibrée:

[Arthur] [mangeait des poissons rouges]

2

6

En substituant "blane" à "rouge", ce qui provoque une collision d'accent et le recul de l'accent de poissons sur la première syllabe, la solution 
MARTIN, Philippe. L'intonation du français et du portugais: phonetique et phonologie.

[Arthur mangeait] [des poissons rouges]

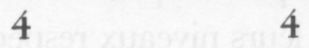

apparaît parfaitement équilibrée.

(Ce mécanisme est utilisé du reste dans une publicité récente pour une bière qui affirme que "cette bière est blanche comme les petits pois sont rouges").

Dans le découpage prosodique précédent, la PS n'est pas congruente à la syntaxe, et cependant c'est la solution qui sera la plus communément réalisée par les locuteurs du français.

Prenons l'exemple

Le frère de Max a mangé les tartines

$\begin{array}{llllllllll}\mathbf{X} & \mathbf{X} & \mathbf{X} & \mathbf{X} & \mathbf{X} & \mathbf{X} & \mathbf{X} & \mathbf{X} & \mathbf{X} & \mathbf{X}\end{array}$

Dont la solution syntaxique d'association d'une structure prosodique paraît relativement bien équilibrée: [2 2] [3 3]. En remplacent "le frère" par "l'arrière arrière grand-père" qui contient cette fois 4 syllabes, et le groupe verbal "a mangé les tartines" par "a disparu", on a

L'arrière arrière grand-père de Max a disparu

$$
\begin{array}{lllllllllll}
\mathbf{X} & \mathbf{x} & \mathbf{X} & \mathbf{X} & \mathbf{x} & \mathbf{x} & \mathbf{x} & \mathbf{x} & \mathbf{x} & \mathbf{X}
\end{array}
$$

Dont la solution eurythmique est cette fois [l'arrière arrière grandpère][de Max a disparu], [6] [6], solution qui viole la contrainte de collision syntaxique, puisque les unités (de Max) et (a disparu) se retrouvent regroupées. La conditions de collision syntaxique l'emporte sur l'eurythmie, et cette réalisation s'avérera mal formée. 
Rev. ANPOLL, n. 6/7, p. 255-295, jan./dez. 1999

Un autre exemple est encore plus révélateur:

Le rhinocéros moyen oriental que tu m'as acheté est perdu

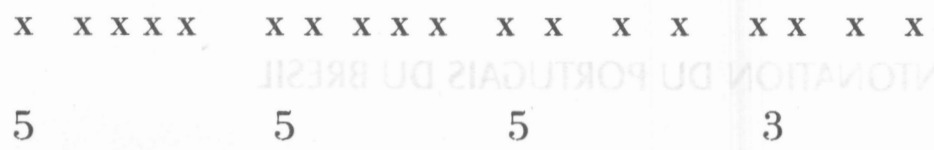

Le conditions de production de l'activité de parole dans le cas de la lecture d'un texte écrit sont particulières. Pour pouvoir en inférer une structure prosodique, il faut faire des hypothèse sur la "qualité" de la lecture, c'est-à-dire la capacité plus ou moins grande du locuteur à lire, donc à analyser la structure syntaxique du texte. Ceci n'est pas une tâche simple, au point qu'elle fasse l'objet d'un apprentissage spécifique au cours des études. En particulier, on peut constater que la capacité à effectuer une analyse syntaxique en plusieurs niveaux hiérarchique varie, et ces variations auront une effet direct sur les structures prosodiques associées au texte. Ainsi certains locuteur limitent systématiquement la profondeur de l'arbre syntaxique à 2 niveaux dans la lecture. D'autres iront jusqu'à 4 , alors que la moyenne semble être de trois niveaux. Ces différences dans la capacité de lecture rend compte de différences observées dans l'intonation de lecture, différences troublantes pour certains phonéticiens.

\section{SOUVERAINETE-ASSOCIATION}

Dans la procédure d'association d'une structure prosodique avec une structure syntaxique donnée, le locuteur doit donc choisir dans la liste de $\mathrm{SP}$ possibles, celles qui ne violent pas la contrainte de collision syntaxique. Parmi les structures retenues, il / elle aura tendance à choisir celle qui est le plus eurythmique, mais ce n'est là qu'une tendance, d'autres considéra- 
MARTIN, Philippe. Lintonation du français et du portugais: phonetique et phonologie.

tions pouvant entrer en jeu dans ce choix, comme la mise en valeur d'une unité particulière etc.

\section{L'INTONATION DU PORTUGAIS DU BRESIL}

$\Lambda$ priori, les principes de description de l'intonation du français relevant des trois fonctions (indication de la modalité, division propos thème et association de la structure prosodique avec la structure syntaxique) s'appliquent aux autres langues romanes, et en particulier au portugais (Martin, 1998, 1999).

En français, le contour de modalité se place sur la dernière syllabe accentuée, qui est en général la dernière syllabe prononcée de la phrase. Si la phrase est divisée en propos et thème, le contour de modalité se trouve sur la dernière syllabe accentuée du propos.

En portugais, la dernière syllabe accentuée n'est pas nécessairement en position finale. La position de contour de modalité est alors la suivante:

1. dans la phrase sans division propos / thème, le contour de modalité déclaratif est positionné sur la syllabe accentuée finale, et le contour interrogatif sur la dernière syllabe (que celle-ci soit accentuée ou non);

2. dans la phrase divisée en propos et thème, les contours de modalité déclaratif et interrogatif sont placés sur la syllabe accentuée finale du propos, et également, mais sous forme neutralisée (donc phonétiquement plate ou faiblement montante) sur la syllabe accentuée finale du thème dans le cas déclaratif, et sur la syllabe finale, accentuée ou non, dans le cas interrogatif.

Il y donc une différence de configuration dans les cas interrogatif et déclaratif, différence non apparente en français du fait de la position nécessairement finale de la dernières syllabe accentuée. 


\section{L'INDICATION DE LA STRUCTURE PROSODIQUE}

Si les caractéristiques théoriques qui régissent les rapports entre les structures syntaxique et prosodique sont valables pour toutes les langues romanes considérées, ainsi que les contraintes propres aux structures prosodiques (collision d'accent, collision syntaxique, eurythmie et longueur maximale d'un mot prosodique), chaque système aura son mécanisme propre, mettant en jeu des marques prosodiques aux réalisations phonétiques spécifiques (tout comme le système phonologique vocalique commun à l'italien et à l'espagnol se voit réalisé différemment sur le plan phonétique).

Considérons une structure prosodique de type $[[\Lambda \mathrm{B}][\mathrm{C} D]]$ raisonnablement susceptible d'être réalisée en lecture soignée comme congruente à une structure syntaxique similaire $((\Lambda \mathrm{B})(\mathrm{C} D))$. Les phrases suivantes sont extraites du corpus Eurom4 (Blanche-Benveniste, 1997), et ont été lues par des locuteurs Portugais et Brésiliens.

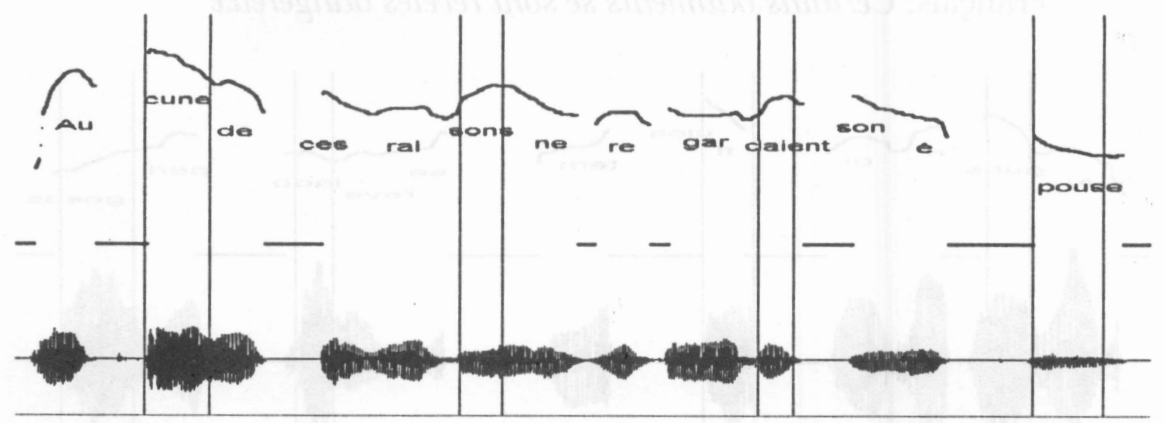

Français: aucune de ces raisons ne regardaient son épouse

(les parties de contour correspondant aux syllabes accentuées sont surlignées). 
MARTIN, Philippe. L'intonation du français et du portugais: phonetique et phonologie.

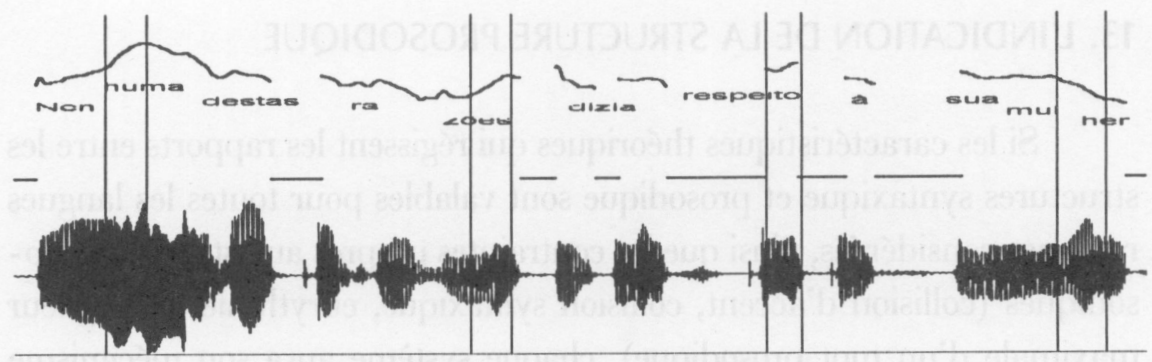

Portugais: Nenhuma destas razões dizia respeito à sua mulher

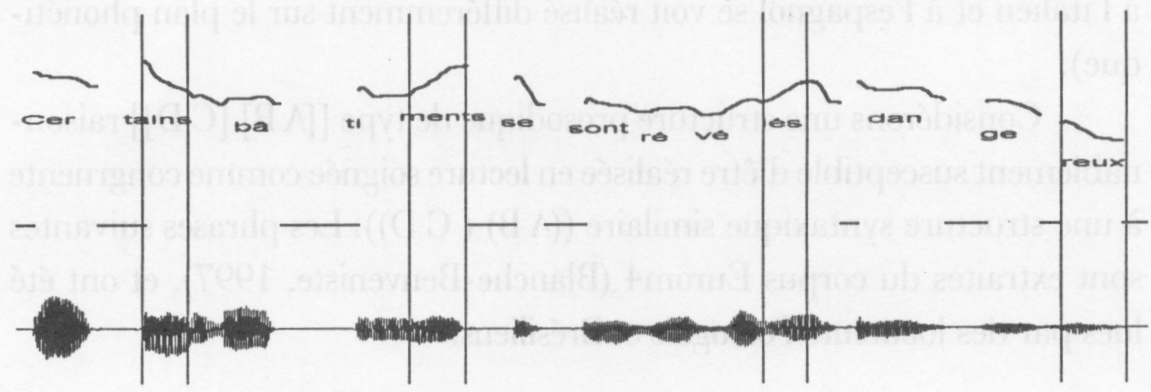

Français: Certains bâtiments se sont révélés dangereux

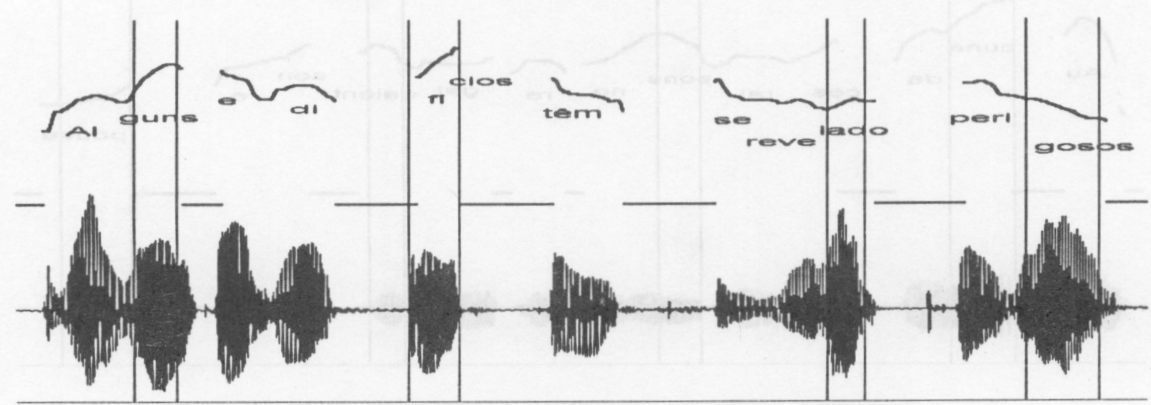

Portugais: Alguns edifícios têm se revelado perigosos

Pour déterminer le mécanisme qui génère les contours, examinons les corrélations de la structure prosodique avec des structures de type (SN 
Rev. $A N P() L L$, n. 6/7, p. 255-295, jan./dez. 1999

SV) et (SP SN SV), en admettant par hypothèse que la congruence prosodie syntaxe est effectivement réalisée dans les données de lecture.

On trouve alors les résultats suivants:

1. Le contour de premier niveau, qui divise la phrase en 2 groupes prosodiques correspondant aux frontières SN SV ou SP SN SV, est manifesté:

a) en français: par une contour montant situé sur la dernière syllabe accentuée de SN. Ce contour est neutralisé est pourra être réalisé par une faible variation mélodique montante ou descendante si SN ne comprend qu'un seul mot prosodique (un seul accent);

b) en portugais: par un système de contours, le premier toujours montant placé (normalement) sur la première syllabe accentuée, un deuxième descendant placé sur la deuxième syllabe accentuée, un contour montant placé sur la dernière syllabe accentuée du syntagme. D'éventuelles syllabes accentuées situées entre la deuxième et la dernière pourront être neutralisée dans leur fonctionnement d'indication de la structure prosodique, et seront alors réalisé par un contour faiblement descendant ou plat.

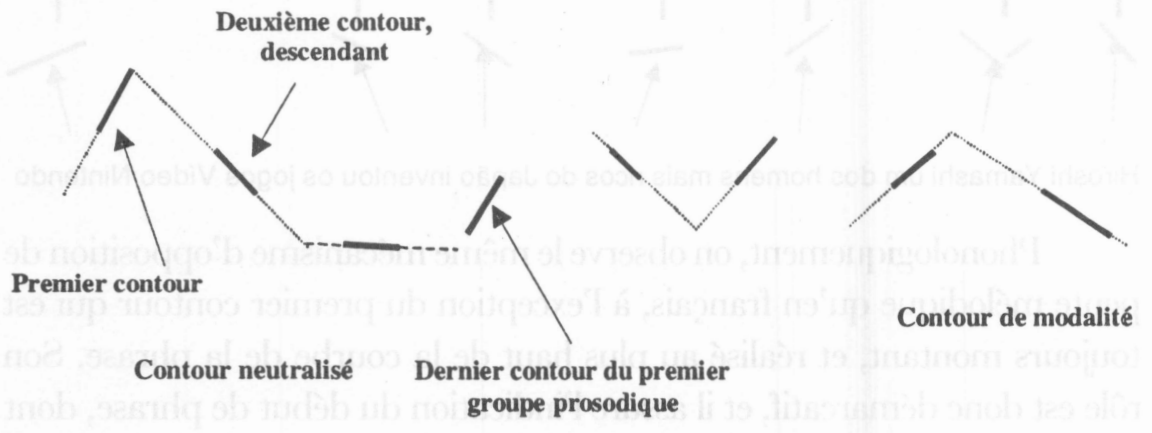

Schéma général des contours de la phrase portugaise. 
MARTIN, Philippe. L'intonation du français et du portugais: phonetique et phonologie.

Ce schéma est réalisé dans l'exemple suivant:

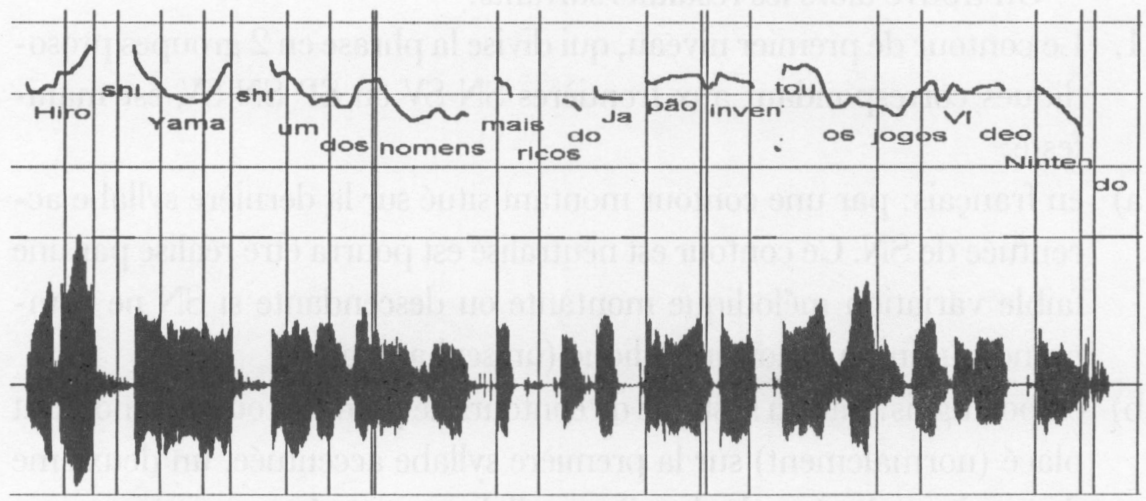

Hiroshi Yamashi um dos homens mais ricos do Japão inventou os jogos Video Nintendo

Si le syntagme ne comporte que deux accents, le dernier contour pourra être descendant montant:

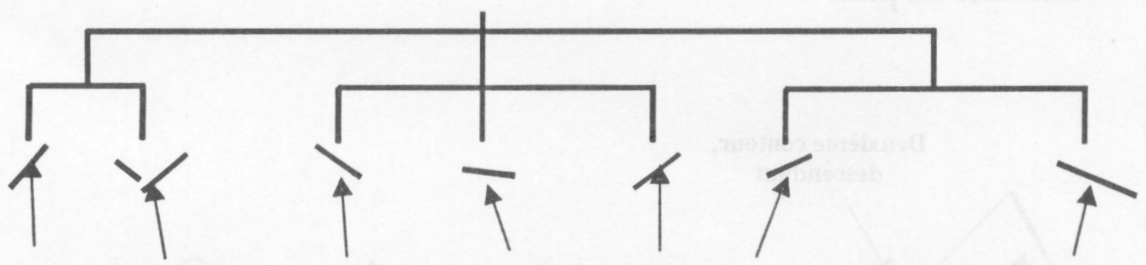

Hiroshi Yamashi um dos homens mais ricos do Japão inventou os jogos Vídeo Nintendo

Phonologiquement, on observe le même mécanisme d'opposition de pente mélodique qu'en français, à l'exception du premier contour qui est toujours montant, et réalisé au plus haut de la courbe de la phrase. Son rôle est donc démarcatif, et il assure l'indication du début de phrase, dont la fin est indiquée par le contour de modalité.

L'examen plus détaillé du premier groupe prosodique permet de déterminer la hiérarchie des contours. 


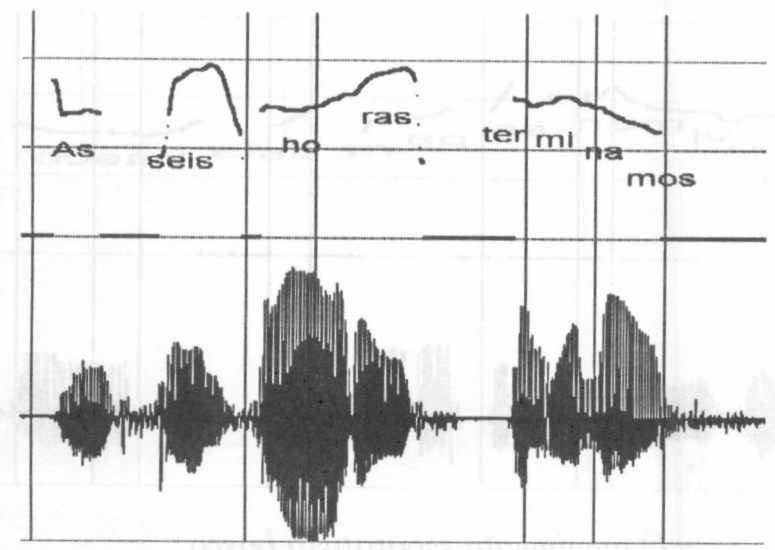

Dans $\grave{s}$ seis horas terminamos, on observe un contour montant haut sur seis creusé bas sur la syllabes accentuée de horas, et montée sur la dernière syllabe, frontière du syntagme. Un autre exemple similaire apparaît ci-dessous:

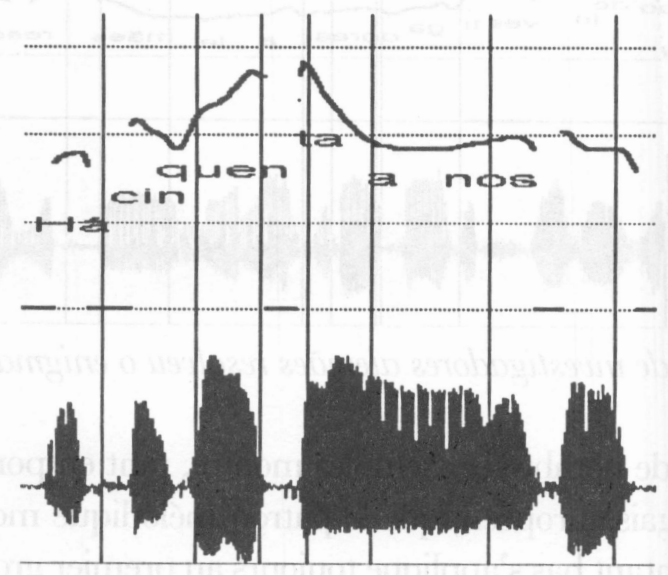

Há cinquenta anos a equipe de Enrico Fermi...

Lorsque le premier groupe prosodique comporte trois syllabes accentuées, le deuxième contour est descendant. 
MARTIN, Philippe. L'intonation du français et du portugais: phonetique et phonologie.

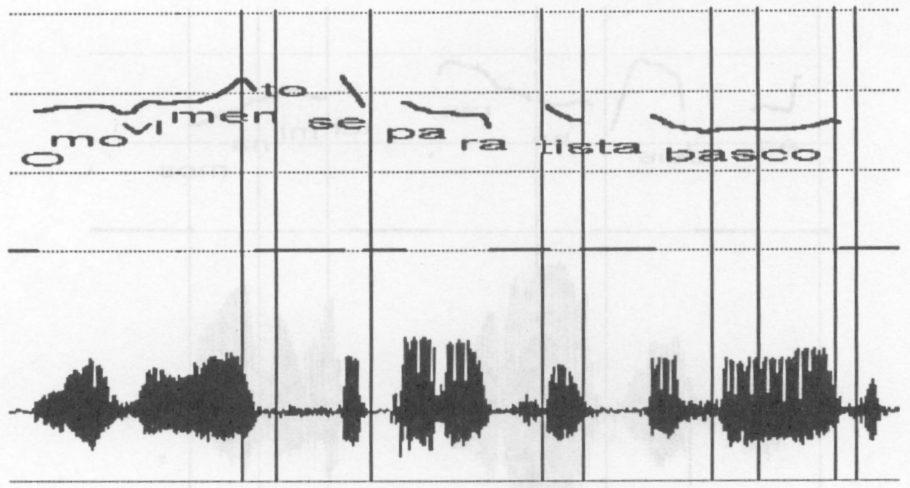

O movimento separatista basco

La même séquence s'observe dans

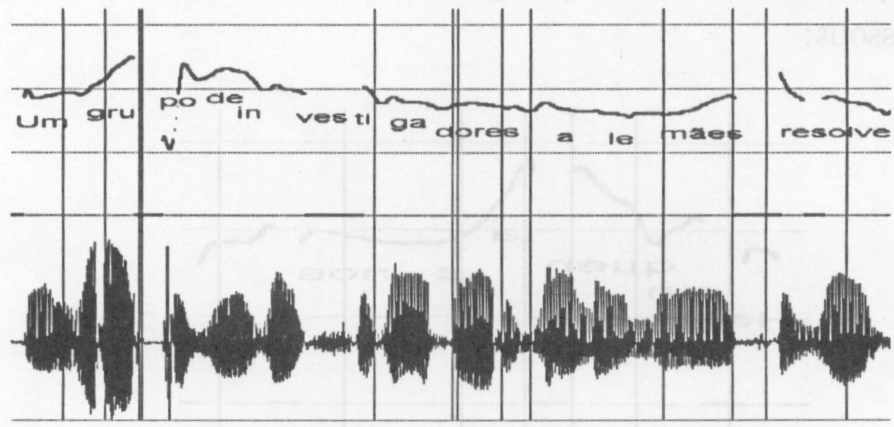

Um grupo de investigadores alemães resolveu o enigma

L'examen de nombreux exemples montre, tant en portugais brésilien qu'en portugais européen, que le patron mélodique montant haut / descendant / montant bas s'applique toujours au premier groupe prosodique. Le dernier contour montant est aligné sur la dernière syllabe accentuée, que celle-ci soit en position finale ou non. Dans ce dernier cas, la syllabe finale non accentuée porte un contour variable, souvent, mais pas obligatoirement, montant. 
Rev. $A N P() L L$, n. 6/7, p. 255-295, jan./dez. 1999

La hiérarchie des contours dans leur fonction indicatrice de structures prosodiques de plus en plus complexe est donc:

Structure à un seul mot prosodique dans le $\mathrm{SN}$ sujet:

Exemple: $O s$ Serbas libertaram cem capacetes azuis

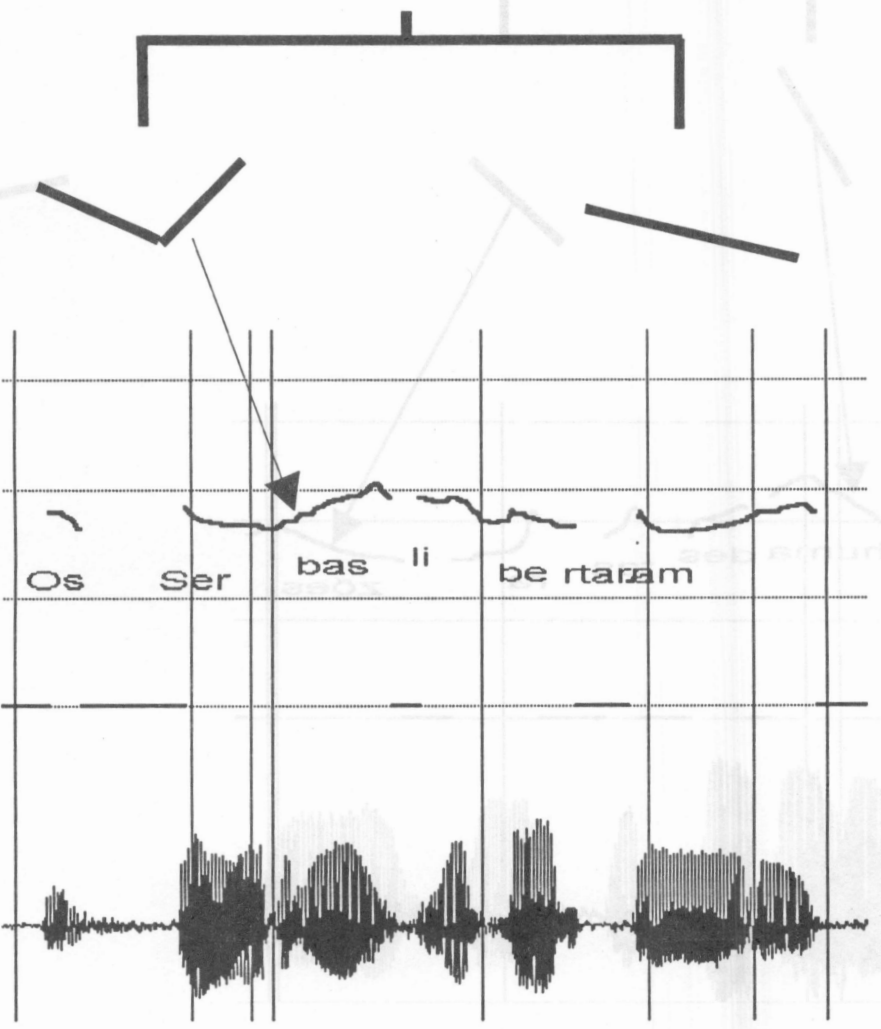

Structure à deux mots prosodiques dans le SN sujet: 
MARTIN, Philippe. L'intonation du français et du portugais: phonetique et phonologie.

Exemple: Nenhuma destas razões dizia respeito à sua mulher

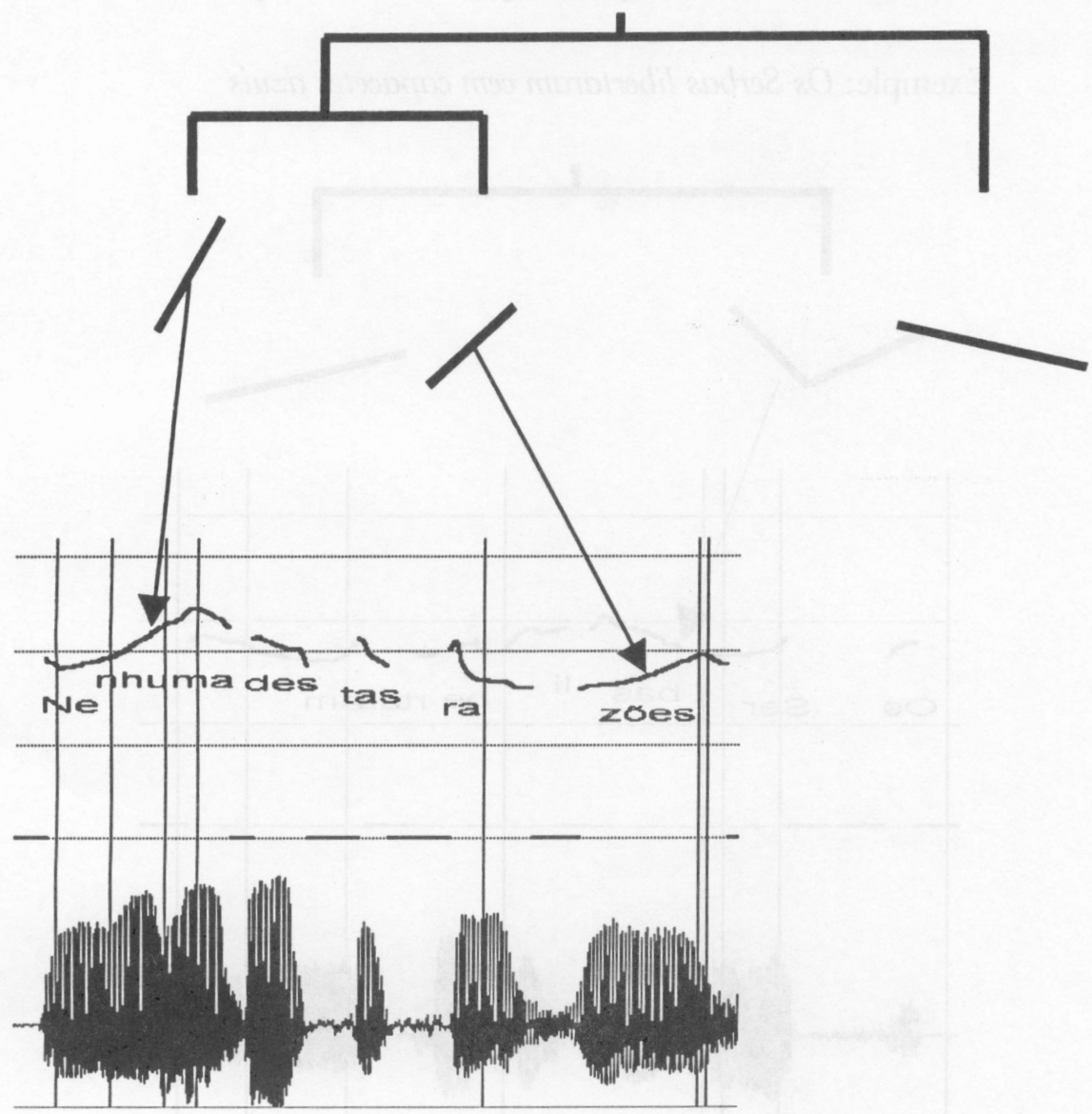


Rev. $A N P() L L$, n. 6/7, p. 255-295, jan./dez. 1999

Structure à trois mots prosodiques dans le SN sujet:

Exemple: Em grupo de investigadores alemães resolveu o enigma

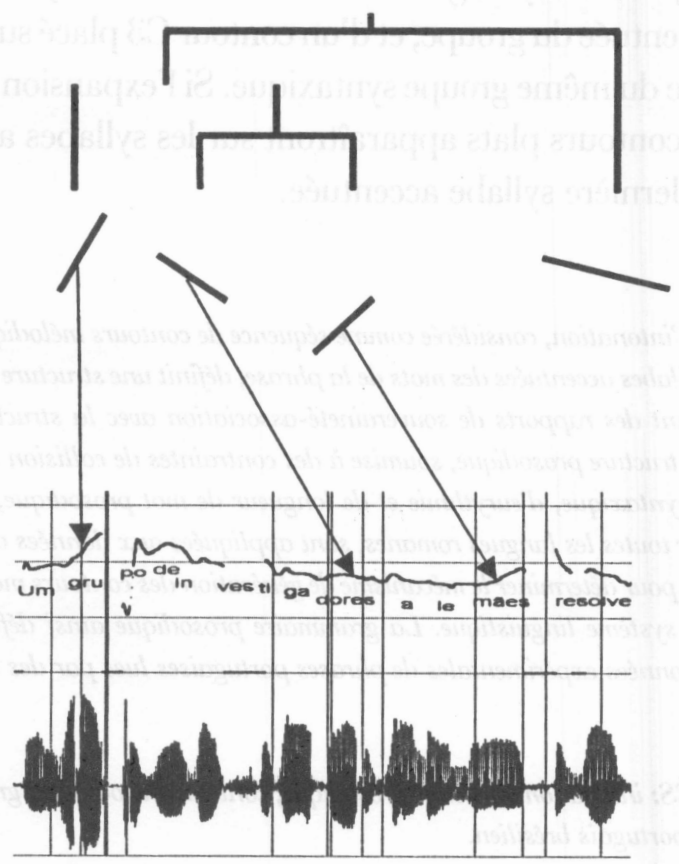

Le système de dépendance des contours prosodiques en portugais s'établit donc comme suit:

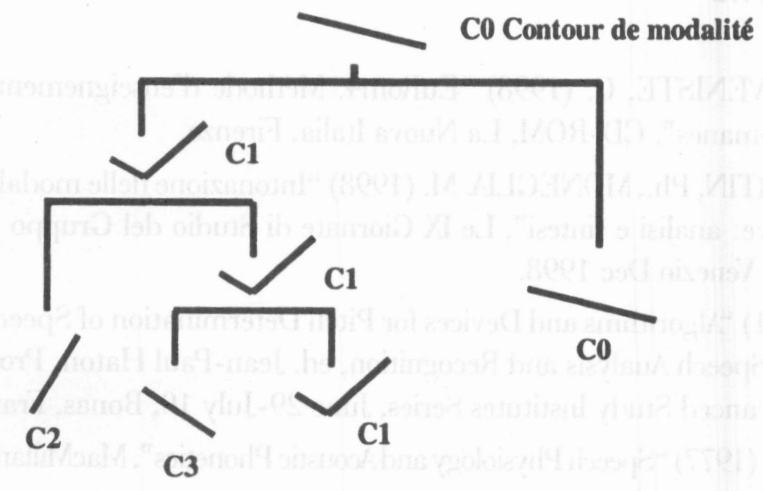


MARTIN, Philippe. L'intonation du français et du portugais: phonetique et phonologie.

Le contour C1 placé sur la dernière syllabe accentuée du premier groupe syntaxique de la phrase présuppose le contour final de phrase CO. L'expansion du premier syntagme révèle le contour C2 placé sur la première syllabe accentuée du groupe, et d'un contour C3 placé sur la deuxième syllabe accentuée du même groupe syntaxique. Si l'expansion du syntagme se poursuit, des contours plats apparaîtront sur les syllabes accentuées du 3ème à l'avant dernière syllabe accentuée.

RÉSUMÉ: L'intonation, considérée comme séquence de contours mélodiques placés sur les syllahes accentuées des mots de la phrase, définit une structure prosodique entretenant des rapports de souveraineté-association avec la structure syntaxique. La structure prosodique, soumise à des contraintes de collision d'accent, de collision syntaxique, d'eurythmie et de longueur de mot prosodique, a priori valables pour toutes les langues romanes, sont appliquées aux données du portugais brésilien pour déterminer le mécanisme de génération des contours mélodiques propres à ce système linguistique. La grammaire prosodique ainsi définie rend compte des données expérimentales de phrases portugaises lues par des locuteurs brésiliens.

MOTS-CLÉS: intonation; structure prosorlique; contours mélodiques; grammaire prosorlique; portugais brésilien.

\section{BIBLIOGRAPHIE}

BLANCHE-BENVENISTE, C. (1998) "EuRom4́. Méthode d'enseignement simultané des langues romanes", CD-ROM, La Nuova Italia, Firenze.

CRESTI, E., MARTIN, Ph., MONEGLIA, M. (1998) "Intonazione delle modalità naturali rappresentative: analisi e sintesi", Le IX Giornate di Studio del Gruppo di Fonetica Sperimentale, Venezio Dec 1998.

HESS, W. J. (1981) "Algorithms and Devices for Pitch Determination of Speech Signals", in Automatic Speech Analysis and Recognition, ed. Jean-Paul Haton, Proceedings of the NATO Advanced Study Institutes Series, June 29-July 10, Bonas, France.

LIEBERMAN, Ph. (1977) "Speech Physiology and Acoustic Phonetics", MacMillan, New York. 
Rev. $A N P() L L$, n. 6/7, p. 255-295, jan./dez. 1999

MARTIN, Ph. (1978) "Questions de phonosyntaxe et de phonosémantique en français", Linguisticae Investigationes, II, 93-126.

MARTIN, Ph. (1983) "Real Time Fundamental Frequency Analysis using the Spectral Comb Method", Proceedings of the Xth Congress of Phonetic Sciences, Foris.

949. . (1987) "Prosodic and Rythmic Structures in French", Linguistics, 25 925(1997) "Sentence Intonation in 4 Romance Languages" in Intonation: theory, Models and Applications, Botinis et al., ed., ESCA, Athens 1997, p. 227-230. . (1998) "Prosodie des langues romanes: analyse phonétique et phonologie", Le français parlé, GARS, Aix-en-Provence, décembre 1998. . (1999) "La musique de la phrase portugaise", en préparation.

WIOLAND, François. (1985) "Les structures rythmiques du français", Slatkine-Champion, Paris.

WINPITCH. (1996-8) "WinPitch, Real-time Speech Analysis", http://www.winpitch.com. 